\title{
Research on the Problems and Management Methods of Radar Equipment in Training Safeguard
}

\author{
Zihua Kong \\ Army Engineering University Shijiazhuang Campus \\ Shijiazhuang, China 050003
}

\author{
Yunwei Zhang \\ Department of Statistics \\ University of California \\ Davis, USA 95616
}

\begin{abstract}
Whether the support for army training is reliable or not is directly related to the win or loss of troops in future battlefield. This paper analyzes and summarizes the problems existing in professional training for troops and equipment support; and deeply explores the countermeasures for constructing support capability of new-type radar equipment in actual combat conditions through research on the current support system of troops and analysis of typical data; then puts forward methods and means for improving support capability of new-type radar equipment to lay a foundation for improving support capability of radar equipment under the condition of actual combat.
\end{abstract}

Keywords-radar; equipment training; talent team construction; equipment safeguade

\section{INTRODUCTION}

With the application of all kinds of new-type radar equipment in troops, the inadaptability of traditional maintenance support mode becomes more and more prominent. Especially, when the troops have old and outmoded equipment and are equipped with maintenance personnel of varying quality, radar equipment support is facing a severe test. When facing the task characteristics of new equipment maintenance support, the maintenance force is not well prepared in support capability and means and their functions are weakened. Therefore, it is an urgent task for the maintenance team to study and solve the problem of how to construct an effective radar equipment maintenance force to adapt to the needs of equipment with new technology and new system and form the support capability of new equipment as soon as possible.

\section{MAIN PROBLEMS IN THE SUPPORT FOR ARMY TRAINING}

\section{A. Shortage of Technical Personnel}

The repair team mainly undertakes medium, minor and support repair in the repair tasks undertaken. And there won't be a problem in the guarantee on repair of old models of radars. However, compared with old models of radars, the new system radars are different from old models on aspects including technical contents, degree of automation, manufacturing technology and working environment which proposes many new requirements on the repair support.
While because the repair team lacks professional technical personnel and dedicated devices, the repair support for new system radar is just limited to fault location and replacement of circuit board; the medium and minor repairs are just limited to the feasible repairs of part of the mechanical parts and electrical equipment but can do nothing to the faults of most of the electrical equipment. The faults of integrated system of new-type radars can only be solved by the manufacturer which will extend the maintenance period and severely impact the serviceability rate of equipment. For the type of investigation radar newly equipped for an armored brigade in 2012, the looseness of power module of computer terminal and the problems of power supply of equipment cause multiple times of faults of equipment within the period more than a month after the allocation and the troop can only seek help to the manufacturer after invalid commissioning. However, because the repairman is not that familiar with the equipment and cannot correctly judge the faults, the three manufacturers of such equipment successively assigned experts to help to repair the equipment which is not only time-consuming but also is labor-consuming and seriously impact the army training.

\section{B. Difficulty in Guarantee of Training Quality}

The first is lack of ideological understandings and lack of enthusiasm for training. Many soldiers think that the guarantee of radar equipment has high requirements on educational background and have the fear of difficulty; some of the soldiers with poor educational background find it hard to learn and cannot thoroughly master it in about a year and cannot easily make achievements; there are also certain soldiers are not willing to learn on their subjective consciousness and some public errand and services occupy the training time; those various kinds of reasons cause it hard to motivate the initiative in training.

The second is lots of equipment types and large difficulties in guarantee. Some units have seven or eight types of radars and one person can only master one or two types of radars but just have a smattering of knowledge about other radars and cannot master other types, therefore the difficulties in guarantee is relatively large. The training organized occasionally just aims at certain type of radar and the training time is short, therefore, the soldiers can only learn very little but cannot thoroughly learn. 
The third is small quantity of personnel. Some units only have one or two radar maintenance personnel and it is hard to develop daily training.

The fourth is small quantity of training equipment and weak practical ability. Most often, the soldiers can only learn along with maintenance which cannot guarantee the usual demands of training and lacks practice opportunities.

\section{Non-synchronous Allocation of Support Facilities}

Currently, new equipment has been gradually equipped in troops, but the allocation of corresponding maintenance support facilities cannot be in position synchronously with the equipment which causes it impossible for some disciplines to develop training and even impacts the repair tasks. There is no maintenance supply prestored in repair teams. Such teams mainly require for repair. At most times, it needs to wait for the allocation of supplier of superior in the middle of equipment repair which directly impacts the efficiency and quality of equipment repair.

Some units have insufficient experiences in emergency maintenance of new radar equipment in actual conditions and lack the ability to conduct battlefield damage assessment to the equipment damaged in battlefield which cause the corresponding emergency maintenance drills to be formalistic and just walk through and this seriously impacts the improvement of guarantee ability for new equipment.

\section{MANAGEMENT METHODS FOR ARMY TRAINING SUPPORT}

\section{A. Enhancing Talent Team Construction}

With the continuous increasing of technical contents of new type equipment, the requirements on the theoretical level and practical operation ability in maintenance support works also become higher and higher. However, there are still the situations existing in many units to different extents where the construction of technical talents for radar repair falls behind the equipment construction development. And the troops mainly rely on manufacturers to solve some difficult technical problem of new equipment which cannot adapt to the requirements on maintenance support in wartime. Therefore, the cultivation of talents must be accelerated from following aspects:

Firstly, select the technical personnel who possess high military and political qualities and strong professional skills and are willing to serve in troop for long term to go to related institutions for further study.

Secondly, the troops shall make efforts in improvement of "three abilities" (management ability of equipment techniques, comprehensive support ability and technological research ability) of technical personnel based on practical conditions and fully exploit the technical potentials of troop based on existing conditions to construct a stable technical backbone team and a new equipment teaching team to actively develop the mutual help and learning activities.

Thirdly, select and send the technical backbone to study in equipment manufacturers or related research institutes or use the opportunity of receiving in manufacturer to conduct targeted intensive training to technical personnel to cultivate the technical talents through multiple channel and multiple levels. The leader of a repair workshop of a troop finds out that the only one technician who can independently accomplish the radar equipment support tasks will transfer to civilian work and go home at the end of year and the radar speciality faces the danger of lack of technicians and the leader of the troop attaches great important to such situation. After collective research, they determine to take full advantage of the opportunities including training in rotation in Wuhan Military Ordnance Sergeant School, the separated training for overall planning within the scope of army group and receiving and delivering, etc and have successively assigned the technical personnel for learning for more than ten person-times within two years which gradually solve the predicament of lack of talents and no talents available.

Fourthly, increase the establishment of senior noncommissioned officers to keep the persons useful for troop equipment support. It can be said that there are certain difficulties in the radar specialty. And it will take a long period to become a "technical expert". Usually, when they can work independently on the technical aspect, they are about to transfer to civilian work and go home. However, the troop establishment is limited and there are no so much ratio of senior noncommissioned officers and the talents are just lost like that which is the greatest loss not matter to the unit or the overall construction of troop. In addition, the hardware and software construction of repair units shall also be continuously enhanced so as to build the good environment favorable for the growth and development of technical talents.

\section{B. Adjusting the Specialty Structure and Proportion}

The scientific and reasonable specialty structure and excellent quality of technical personnel are essential to guarantee the equipment maintenance quality and the issues on the two aspects must be well solved. For example, the professional technical talents in troops of western developed countries account for $30 \%$ to $40 \%$ in troops and account for up to $70 \%$ to $80 \%$ in the technology-intensive departments, while China has a certain difference on this aspect. Although the existing specialties in repair teams have been developed to be relatively mature, they are still not reasonable enough when facing the maintenance support for new system equipment. A repair troop has the normal establishment of radar repairman of four persons including a technician, two repairmen and a driver and repairman. After the establishment system adjustment, the tasks are increased and not only the medium and minor repair tasks shall be accomplished in a year, it shall also support the repair of the units under support, therefore, the personnel are obviously in shortage and it becomes much more difficult in the support and it will be inevitable to catch one and lose another. Therefore, it is suggested that all repair units may suitably increase the establishment quantity of radar repair personnel based on the existing establishment and according to the actual task conditions. For the technical personnel not in establishment, except for cultivation of units, it can also 
transfer the technical personnel with strong professional skills and strong sense of responsibility from the basic level to the repair troop. The allocation of repairmen shall guarantee that there shall be at least one or two technical experts leading for each type of radar through supplementing the shortage according to the guarantee requirements and shall guarantee the good situation with multiple repairmen participating in the support. Only in this way can we guarantee to form a mobile emergency repair support team at any time in wartime to implement supporting emergency repair in war zone or larger range.

\section{Improving the Training Support System}

The fundamental reason for inadequate attention to and implementation of professional training lies in the lack of power to train troops and the failure to motivate troops to focus on and carry out training, thus resulting in indefinite training goals, lack of standardization in organizing training and impeded running of mechanisms. To implement supporting training, first, leaders shall play a leading role in practicably establishing the awareness that the supporting training serves as an important link for ensuring equipment support and a significant means for cultivating technical force. Leaders and cadres shall take charge of general classes, manage key classes and participate in difficult classes all by themselves. Second, planning shall be comprehensive. Attention shall be paid to the teaching and training capacity of trainers, thus improving the capacity of the whole troop. Training shall be summarized and improved to find out deficiencies and then lay a solid foundation of professional training. Third, a reward and punishment mechanism shall be established, and great emphasis shall be put on the work to which the mechanism applies. Training effect shall be linked to benefits, be under the charge of all relevant personnel and apply to the implementation of reward and punishment, thus solving the problem of treating equally irrespective of training effects. Daily test, weekly appraisal and monthly reward system shall be carried out to test those who have participated in training, promote those who are eligible in tests and reward those who win. However, those who fail to complete the training tasks, gain little in the mentoring group and interfere in the training fulfillment shall be held responsible.

\section{Strengthening the Construction of Supporting Facilities for Maintenance Equipment}

Since new equipment has high technology content and high integration of components, some old maintenance equipment has failed to adapt to new demands for maintenance support. Thus, the following three aspects of work shall be done to strengthen the construction of supporting facilities for maintenance equipment.

Firstly, great attention shall be paid to the reliability of the support facilities for new equipment and their adaptability to battlefields. Support facilities and operation equipment shall be developed synchronously. That is, supporting support facilities shall be developed in accordance with the development plan for equipment in the new system, and shall be timely provided to troops. However, following blindly is forbidden. In order to avoid this, omissions and deficiencies shall be found and solved in the practice of equipment support training, thus improving the adaptability of support facilities and equipment to battlefields.

Secondly, emphasis shall be put into the integral development of new and old support equipment. By giving a full play to the role of old maintenance support equipment, intensity of technological reforms shall be increased to improve its maneuverability, safety protection ability and automated maintenance ability. Since the whole replacement of some facilities and equipment requires a long period, it is more practical in terms of the cost performance to improve the performance of old equipment through update and innovation.

Thirdly, the development dynamics of new technologies and support facilities in the new system shall be timely followed up. Based on respective comprehensive demands for support tasks and support equipment, reasonable construction plans for support equipment shall be made to gradually equip new equipment with supporting maintenance support facilities. Equipped facilities shall conform to the principles of integration, digitalization, generalization, miniaturization and modularization, being convenient to carry out repair near the front lines and thus adapting themselves better to the needs in actual combats.

\section{E. Formulating a Security Plan That Is Close to Actual Combat}

As a technician in the front line of repair, it is necessary to start from the most complicated situation, fully consider the requirements for wartime equipment support, and formulate radar repair plans in complex situations such as multi-level and multi-regional. Four aspects should be fully taken into account in the plan: the first is the prediction of battle-damaged equipment and the method of emergency repair, the alternative scheme of large-scale battle-damaged components, the configuration and supplementary scheme of support equipment, the communication and liaison command mode under various circumstances, etc.; the second is the supply channel of emergency equipment, apparatus repair and maintenance materials; the third is the topography, natural resources, social environment and traffic conditions around the station; the fourth is that the emergency repair drills shall be often carried out. It's necessary to simulate the radar equipment support training drills in the actual combat environment, so as to improve the comprehensive emergency response ability of repairmen.

\section{CONCLUSION AND SUGgestion}

With the continuous renewal and development of new radar equipment, the contradiction between equipment support capability and support training needs has become increasingly prominent. In order to solve this contradiction, it's essential to do the following: 


\section{A. Training Qualified Personnel}

First, it is important to grasp the training situation and seize the opportunity to do practices. The task of the repair unit mostly concentrates on the period of professional training. In the training time of common subjects and basic subjects, the duty of repair and maintenance is relatively light, which is the best time for the maintenance unit to strengthen training and improve the repair level.

Secondly, it's crucial to intensify the cultivation of compound talents, strengthen the cultivation of technical personnel's capability of "one specialty, multi-ability and multi-specialty, multi-ability" to give full play to the greatest effectiveness of talents.

Thirdly, it's essential to attach importance to the fostering of novices. Talent training is no crash course. At present, it's good for the training time to be arranged for lasting more than one month but less than two months by considering that for the newcomer, it is difficult to have a pretty grasp in a short time and easy to be distracted in a long time. To master a new kind of equipment, a repairman of above the junior level needs more than eight months 'training or many times' training to reach a certain level of support ability.

In practice, we should boldly use new people and "make the senior teach newcomers". Even if newcomers occasionally make some small mistakes in maintenance support (for example, damage small parts, etc.), leaders should treat them with tolerance and should not severely criticize them. Otherwise, they will lose their enthusiasm for maintenance support. Only through continuous practice and training can newcomers become professional backbone. Most of the technical backbones of the repairing units have already got married. Leaders at all levels should take the initiative to tackle problems for these new equipment maintenance support personnel, solve their worries, make sure to retain talents and their hearts, and strive to create a good environment for them. Only in this way can technical personnel take service at ease and devote all their energy to the construction of new equipment support.

\section{B. Close to the Actual Combat}

In the training of equipment support, we should change our concepts and try our best to simulate the requirements to start training. We should take the training ground as the first battlefield, make full use of opportunities of military exercises and field training, strengthen the content of equipment support training, and train the equipment support capability of our troops in field operations. We can also carry out regular itinerant repairs within the scope of the group army, so that the repairmen in various units can learn from each other regularly, jointly enhance their professional level, and improve the equipment support capability of the army in practice. Besides, we should take full advantage of the army's winter training, further explore and study the radar equipment support work in cold conditions, and exercise the equipment support ability in field operations. We can test the reliability, timeliness and accuracy of army equipment support through support training in the actual combat environment.

\section{REFERENCES}

[1] Yu Jiasheng, Huang Yunhai, Yao Zhilong. Research on Wartime Equipment Guarantee Law. (in Chinese)

[2] Sun Hui, Sun Likun, Jiang Yupu: Research on the Construction of Operational Support Capability of New Ordnance Equipment. (in Chinese)

[3] The Outline of Military Training and Evaluation for the Army (Ordnance Equipment Support Team). (in Chinese) 